\title{
Effect of Polarity and Rise Time of Applied Pulsed Voltage on Streamer Discharge Phenomena
}

\author{
K. Yoshinaga, S. Okada, D. Wang, T. Naminira*, S. Katsuki and H. Akiyama \\ Graduate School of Science and Technology, Kumamoto University \\ 2-39-1 Kurokami, Kumamoto 860-8555, Japan
}

\begin{abstract}
Pulsed streamer discharges have been extensively used in many applications such as control of $\mathrm{NO}_{X}$ and $\mathrm{SO}_{2}$ from exhaust gases, treatment of dioxins, removal of volatile organic compounds, generation of ozone and laser excitation. An operation with a high-energy efficiency is necessary for practical applications. It is very important to know the propagation mechanism of streamer discharges in order to improve the energy efficiency of pulsed discharge systems. In this paper, the emission from pulsed streamer discharges in a coaxial electrode system in air at $0.1 \mathrm{MPa}$ was observed using a high-speed gated intensified charge-coupled display camera. A concentric wire-cylinder electrodes configuration was used. Positive and negative pulsed voltages having a width of about $200 \mathrm{~ns}$ were applied to the central electrode. From the results, the streamer discharges were initiated at the inner electrode and terminated at the outer electrode. It is suggested that the propagation velocity of the streamer discharges is being influenced strongly by the rise time and the polarity of the pulsed voltage.
\end{abstract}

PACS numbers: $52.80 . \mathrm{Hc}$

\section{Introduction}

Non-thermal plasma has been one of the promising technologies for the removal of hazardous environmental pollutants in gases. A pulsed streamer discharge in atmospheric pressure gases is one type of non-thermal plasma and has received a great deal of interest for many years. The critical factor to enable this technique for industrial application of pollutant removal is to improve the energy efficiency of the plasma processing. For the improvement of the energy efficiency, it is necessary to know the propagation mechanism of the pulsed streamer discharges.

In this paper, the detailed propagation characteristics of a pulsed streamer discharge produced by the different polarity and the different rise time of pulsed voltage application are reported. The pulse width of the applied voltage was fixed at $200 \mathrm{~ns}$ and the coaxial electrode filled with atmospheric pressure air. The propagation process and the velocity of the streamer head observed by a high-speed gated intensified ICCD camera was reported in this work.

\section{Experimental apparatus and procedure}

A schematic diagram of the experimental arrangement was same to $[1,2]$. A Blumlein line generator with a pulse width of $200 \mathrm{~ns}$ and a pulse transformer (PT) were used as a pulsed-power supply in this paper. For the

* corresponding author; e-mail: namihira@cs.kumamoto-u.ac.jp operation of the pulsed-power supply, the Blumlein generator was charged up to $30 \mathrm{kV}$, and the output voltage from the Blumlein generator was stepped up by PT. The winding ratio of the $\mathrm{PT}$ was fixed at 1:3. To change the rise time of the pulsed voltage, the different winding number of the primary and secondary windings of the PT was used under the same PT winding ratio of 1:3. Typically, the rise time of the pulsed voltage was $20 \mathrm{~ns}$ when the primary and secondary winding numbers were 1 turn and 3 turns, respectively. In the case of the PT winding numbers of 3 and 9 turns, the rise time of $64 \mathrm{~ns}$ was generated. The both polarities of the positive and the negative of the pulsed voltage were obtained by the changing the ground connection of the PT. A coaxial cylindrical chamber was utilized as a discharge electrode to observe a pulsed streamer discharges. A canter electrode made of stainless steel having $0.5 \mathrm{~mm}$ in diameter and $10 \mathrm{~mm}$ in length was placed concentrically in copper cylinder. The outer cylinder electrode was grounded, and its diameter was $60 \mathrm{~mm}$. The chamber was filled with dry air at $0.1 \mathrm{MPa}$. A high-speed gated ICCD camera (C7972-01, Hamamatsu Photonics, Japan) with MCP (Micro Channel Plate, maximum gain $=10000$ ) was used to observe the discharge emission from streamer discharges. The exposure time was fixed at 200 ps. The delay time after application of voltage was varied up to $300 \mathrm{~ns}$.

\section{Results and discussion}

Figure 1 shows the typical waveforms of the applied voltage and the discharge current in the electrode gap, and the images of emissions from discharges as a function 


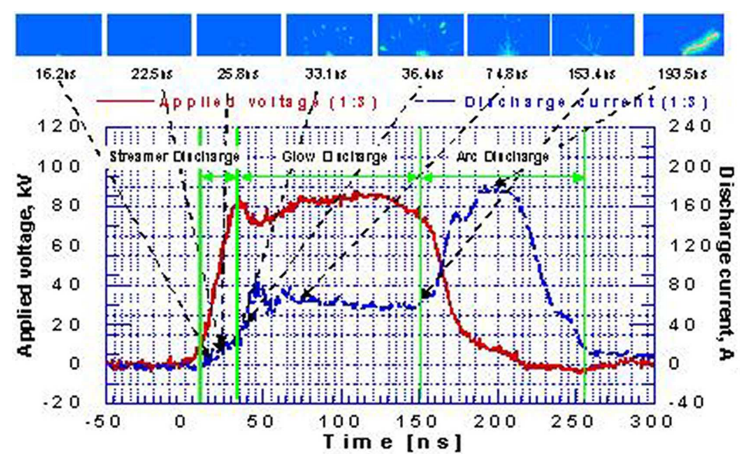

Fig. 1. Typical time dependences of the applied voltage to and the discharge current through the discharge chamber, and the discharge image taken by ICCD camera. (Positive polarity, PT's winding number of primary and secondary $=1: 3$.).

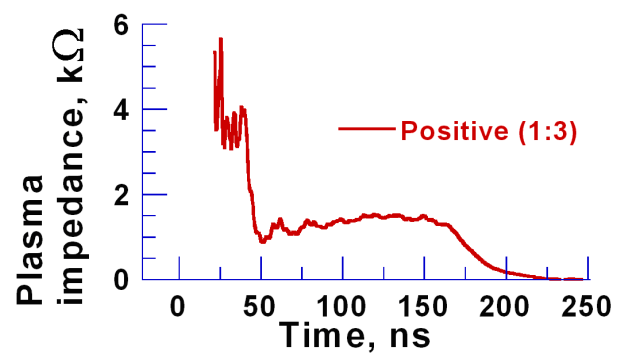

Fig. 2. Time dependence of discharge impedance calculated for Fig. 1.

of time after the initiation of the pulsed voltage in the case of the application of the positive pulsed voltage with fast rise time. Figure 2 shows the time dependence of the discharge (electrode) impedance calculated from the voltage and current waveforms in Fig. 1. It is observed from Fig. 1 that the streamers propagate from the central to the outer electrodes and the discharge current was small during the streamer propagation. After the fully development of the streamer discharges, the discharge phase transformed to the glow-like discharges from the streamer

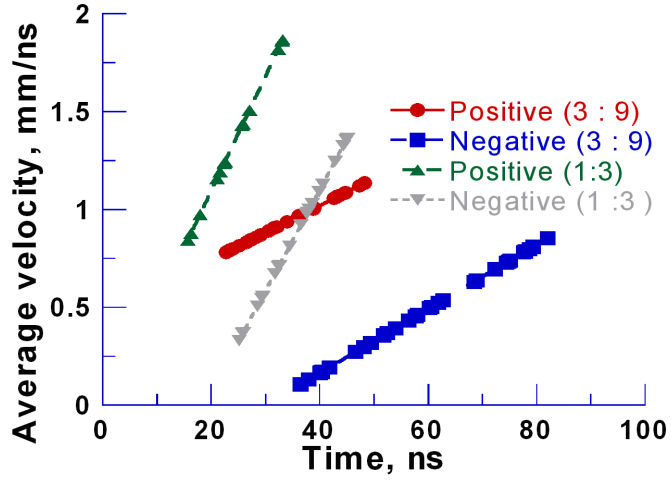

Fig. 3. Time dependences of average velocities of streamer heads for all discharge conditions.

discharge. During the glow-like discharge phase, the discharge current was kept around 35 A. After 110 ns of glow-like discharge phase, the discharge phase, finally, transformed to the arc discharge with rapid increase of the discharge current. It can be seen in Fig. 2 that the discharge impedances of the streamer, glow-like and arc discharges were kept at $3.5 \mathrm{k} \Omega, 1.2 \mathrm{k} \Omega$ and $0 \Omega$, respectively. It might be thought that the discharge impedance has strong influence on the temperature of plasmas due to the discharge [3].

Figure 3 shows the dependences of the average velocity of the streamer heads on the time after the pulsed voltage application for all discharge conditions. The average velocity of the streamer heads is given by the following equation:

$$
V_{\text {streamer }}=\frac{L}{t_{\text {delay }}},
$$

where $L$ and $t_{\text {delay }}$ are the distance between the surface of the inner electrode and the tip of streamer head and the delay time after the pulsed voltage application, respectively. It is observed from Fig. 3 that the velocity of the streamer heads increased with increasing time for all discharge conditions.

TABLE

Summary of discharge phenomena for all discharge conditions.

\begin{tabular}{|c|c|c|c|c|}
\hline Polarity of applied pulsed voltage & Posit & tive & Nega & itive \\
\hline PT's primary and secondary winding number & $1: 3$ & $3: 9$ & $1: 3$ & $3: 9$ \\
\hline maximum voltage, $\mathrm{kV}$ & 84 & 88 & -106 & -103 \\
\hline rise time of applied pulsed voltage (10 to $90 \%$ ), ns & 20 & 64 & 25 & 59 \\
\hline rise rate of applied pulsed voltage (10 to $90 \%$ ), $\mathrm{kV} / \mathrm{ns}$ & 3.3 & 1.1 & 3.4 & 1.4 \\
\hline duration of streamer discharge, ns & 34 & 50 & 41 & 81 \\
\hline average velocity of streamer heads, $\mathrm{mm} / \mathrm{ns}$ & $1.88-0.84$ & $1.14-0.78$ & $1.38-0.34$ & $0.85-0.10$ \\
\hline average acceleration of streamer heads, $\mathrm{mm} / \mathrm{ns}^{2}$ & 0.059 & 0.014 & 0.052 & 0.016 \\
\hline impedance of streamer discharge, $\mathrm{k} \Omega$ & 3.6 & 7.1 & 4.7 & 8.9 \\
\hline duration of glow-like discharge, ns & 110 & 52 & 97 & 44 \\
\hline impedance of glow-like discharge, $\mathrm{k} \Omega$ & 1.2 & 1.2 & 1.2 & 1.2 \\
\hline transient time to arc discharge from pulsed voltage application, ns & 160 & 134 & 138 & 147 \\
\hline
\end{tabular}


The speed of the streamer developed by the positive applied voltage is greater than that by the negative one. In both polarities of the applied voltage, the streamer velocity becomes fast with faster rise time of the applied voltage.

Table shows the summary of discharge phenomena for all discharge conditions. In Table, impedance of streamer discharge becomes greater with faster rise time of the applied voltage in both polarities of the applied voltage. However, impedance of glow-like discharge was equal for all discharge conditions. From Table it can be seen that, in both polarities of the applied voltage, duration of glow-like discharge becomes longer with faster rise time of the applied voltage.

\section{Conclusions}

Discharge phenomena by the pulsed voltage application having the different polarity and rise time were observed by the high-speed gated ICCD camera in the work. The conclusions are below:
1) During the pulsed discharge, the discharge phase transformed from streamer to glow-like and finally to arc.

2) The polarity and the rise time of pulsed applied voltage has strong influence on the average velocity of the streamer heads during streamer discharge.

\section{References}

[1] T. Namihira, D. Wang, S. Katsuki, R. Hackam, H. Akiyama, IEEE Trans. Plasma Sci. 31, 1091 (2003).

[2] T. Namihira, T. Tokuichi, H. Tamaribuchi, D. Wang, S. Katsuki, H. Akiyama, Proc. of 10th International Symposium on High Pressure, Low Temperature Plasma Chemistry, Saga, Japan, 17 (2006).

[3] D. Wang, M. Jikuya, S. Yoshida, T. Namihira, S. Katsuki, H. Akiyama, IEEE Trans. Plasma Sci. 35, 1098 (2007). 